\title{
1 Novel Computational Method to Define RNA PSRs Explains Influenza A Virus 2 Nucleotide Conservation
}

3 Andrey Chursov ${ }^{1}$, Nathan Fridlyand ${ }^{2,3}$, Albert A. Sufianov ${ }^{4,5}$, Oleg I. Kiselev ${ }^{6,}{ }^{*}$, Irina

4 Baranovskaya $^{6}$, Andrey Vasin ${ }^{6}$, Jonathan W. Yewdell ${ }^{3}$ and Alexander Shneider ${ }^{5,7}$

$5 \quad{ }^{1}$ Datomics Group, 71 Amalienstr., Munich, 80799, Germany

$6{ }^{2}$ Laboratory of Translational Biology, School of Biosciences and Biotechnology, University of

7 Camerino, Via Gentile da Varano III, 62032, Camerino, Italy

$8{ }^{3}$ Cellular Biology Section, Laboratory of Viral Diseases, NIAID, NIH

$9{ }^{4}$ Federal Center of Neurosurgery of the Ministry of Health of Russian Federation, 5

10 Chervishevskiy Trakt, Tyumen, 625062, Russia

$11{ }^{5}$ I.M. Sechenov First Moscow State Medical University, 8 Malaya Trubetskaya St., Moscow, 12 119048, Russia

$13{ }^{6}$ Smorodintsev Research Institute of Influenza, Prof. Popova str. 15/17, Saint-Petersburg,

14 197376, Russia

15 *deceased

$16{ }^{7}$ Department of Molecular Biology, Ariel University, Ariel, Israel

18 Email addresses:

19 AC: achursov@datomicsgroup.com

20 NF: snatanf@gmail.com

21 AAS: info@fcn-tmn.ru

22 OIK: oleg kiselov@hotmail.com

23 IB: irina.baranovskaja.1992@gmail.com

24 AV: influenza.spb@gmail.com

25 JWY: jyewdell@nih.gov

26 AS: ashneider@curelab.com

\section{ABSTRACT}

RNA molecules often fold into evolutionarily selected functional structures. Yet, the literature offers neither a satisfactory definition for "structured RNA regions", nor a computational method to accurately identify such regions. Here, we define structured RNA regions based on the premise that both stems and loops in functional RNA structures should be conserved among RNA molecules sharing high sequence homology. In addition, we present a computational approach to identify RNA regions possessing evolutionarily conserved secondary structures, RNA ISRAEU (RNA Identification of Structured Regions As

36 Evolutionary Unchanged). Applying this method to H1N1 influenza mRNAs revealed

37 previously unknown structured RNA regions that are potentially essential for viral replication 
and/or propagation. Evolutionary conservation of RNA structural elements may explain, in part, why mutations in some nucleotide positions within influenza mRNAs occur significantly more often than in others. We found that mutations occurring in conserved nucleotide positions may be more disruptive for structured RNA regions than single nucleotide polymorphisms in positions that are more prone to changes. Finally, we predicted computationally a previously unknown stem-loop structure and demonstrated that oligonucleotides complementing the stem (but not the loop or unrelated sequences) reduce viral replication in vitro. These results contribute to understanding influenza A virus evolution and can be applied to rational design of attenuated vaccines and/or drug designs based on disrupting conserved RNA structural elements.

\section{AUTHOR SUMMARY}

RNA structures play key biological roles. However, the literature offers neither a satisfactory definition for "structured RNA regions" nor the computational methodology to identify such regions. We define structured RNA regions based on the premise that functionally relevant RNA structures should be evolutionarily conserved, and devise a computational method to identify RNA regions possessing evolutionarily conserved secondary structural elements. Applying this method to influenza virus mRNAs of pandemic and seasonal H1N1 influenza A virus generated Predicted Structured Regions (PSRs), which were previously unknown. This explains the previously mysterious sequence conservation among evolving influenza strains. Also, we have experimentally supported existence of a computationally predicted stem-loop structure predicted computationally. Our approach may be useful in designing live attenuated influenza vaccines and/or anti-viral drugs based on disrupting necessary conserved RNA structures.

\section{INTRODUCTION}

The biological functions of RNA secondary structures and their evolutionary impact is a topic of great interest and importance (1-11). Since 1990s, conceptually novel computational approaches have been appearing to analyze RNA shapes. Multiple copies of the same RNA molecule fold into different coexisting conformations constituting an ensemble of RNA structures. The same nucleotide within the RNA may be coupled via W-C bonds in some conformations (being a part of a stem) while remaining uncoupled in others (i.e. belonging to a loop). If one analyzes the entire ensemble, they can attribute to each nucleotide within an RNA sequence its base pairing probability. This probability value reflects i) the percentage of RNA structures which have this particular nucleotide W-C bonded (although in different structures it may be bonded to a different coupling partners), and ii) the likelihood of each RNA structure within the ensemble of all possible conformations that is based on the structure's free energy. Hence, if an RNA molecule contains $X$ nucleotides, a series of $X$ numbers ranging from 0 to 1 can be estimated such that each number reflects the probability of a nucleotide in this position to be coupled. 
79 Viruses represent an excellent system for studying RNA structural biology based on several

80 factors, including the high abundance of viral RNAs, the high mutation rate of many viruses,

81 the ease of selecting conditional mutants, and the large number of closely related strains

82 available for sequence conservation analysis.

83 Mutations disrupting influenza A virus RNA secondary structures dramatically reduce the 84 levels of gene expression (12). We previously reported that influenza A virus, a negative 85 strand RNA virus with a segmented genome, possesses clusters of nucleotides that 86 significantly change their base pairing probabilities with temperature elevation (13). This 87 suggests that local structures dispersed between non-structured RNA regions (nonPSRs) 88 are evolutionarily selected.

89 Previous attempts to define "structured RNA regions" were aimed either at finding regions 90 possessing the most stable secondary structure predicted by minimum free energy (MFE) of 91 based paired sequences (14-16), or predicting a consensus secondary structure based on a 92 given multiple-sequence alignment, which can be inferred either by means of energy93 directed folding or using a phylogenetic stochastic context-free grammar model (17-21). The 94 latter approach works well on short non-coding RNAs, which usually have one predicted stable structure, but its accuracy drops significantly with the increasing RNA length (22). In addition, mRNAs are typically less structured than non-coding RNAs, since structures interfere with translation by ribosomes (23). At the same time, the former approach misinterprets "PSRs" as stems possessing abnormally many W-C coupled nucleotides; i.e. it would view an RNA structure possessing evolutionarily conserved loops (where the

100 nucleotides are not paired) as an unstructured element. The following example

102 a cloverleaf-like secondary structure may serve an indispensable biological function and be

103 conserved in every strain of some organism, despite the fact that it may have fewer paired

104 nucleotides than a simple stem (see Figure 1). Also, such approach may introduce systemic

105 bias typically identifying mRNAs as less structured than non-coding RNAs, since excessive

106 abundance of W-C pairing may interfere with translation by ribosomes (23).

107 In addition, any particular RNA sequence beyond certain length clearly can fold into stable

108 alternative states with energies being somehow different from the global minimum (24-26);

109 and several alternative RNA conformations coexist at equilibrium (27). Some of these RNA

110 structures may be present in multiple RNAs, especially homologous ones, while others

111 would exist for the particular sequence only. One can assume that evolutionary conservation

112 of the RNA shape may be indicative of its biological function. Current approaches relied

113 upon analysis of a single sequence cannot differentiate an evolutionarily conserved

114 structural element from an RNA shape that is energetically favorable only in a particular

115 strain. Thus, future progress in the field requires a new methodology. Addressing these

116 problems and proposing a computational methodology free of these shortcuts is the main

117 aim of this paper.

\section{Results}

\section{A Novel Quantitative Definition of Structured RNA Regions}


121 Here, we present a quantitative definition of a structured RNA region (PSR) that is equally

122 useful to predicting both, stems and loops as structured regions based on their evolutionary

123 conservation, and a new computational method for identifying those regions. The method is

124 robust to random RNA shapes present in a particular sequence but not selected and

125 conserved evolutionarily. We call this method RNA ISRAEU (RNA Identification of Structured

126 Regions As Evolutionary Unchanged).

127 As the first step in this method, we created a non-redundant dataset of sequences for each

128 RNA of interest constituted of highly homologous RNAs of the same length, and built

129 multiple sequence alignments. For the second step, the probability of every nucleotide to be

130 paired was calculated for each RNA sequence in the dataset. Third, we substituted every

131 nucleotide in the multiple alignment, with the nucleotide's pairing probability, thus aligning

132 pairing probabilities by nucleotide position. We took probabilities to be paired for all the 1st

133 nucleotides in each RNA sequence and grouped them together; for all $2^{\text {nd }}$ nucleotides; for all

$134 \mathrm{~N}^{\text {th }}$ nucleotides. Thus, if we have $X$ RNA sequences each constituted of $Y$ nucleotides, we

135 create $Y$ sets of numbers ranging from 0 to 1 ; each set contains $X$ numbers. Standard

136 deviation was computed for each set of probability values corresponding to every position

137 within the RNA. We proposed that such standard deviations be used as a measure of

138 structural conservation in a specific position. If the standard deviation for a particular position

139 within the RNA dataset was small, the probability of a nucleotide to be in a double-stranded

140 conformation did not vary substantially across the entire dataset of aligned mRNAs. We call

141 such positions "structure-conserved". In contrast, if the standard deviation was high, the

142 probability of a nucleotide to be paired changed vastly from strain to strain, a position was

143 called "structure-variable". The mean probability at a particular position did not matter to the

144 position classification.

145 We called regions within RNA sequences formed by consecutive structure-conserved 146 positions "Predicted Structured Regions" (PSRs), while regions predominantly formed by 147 structure-variable positions were called "non-structured" (nonPSR). Apparently, such 148 definition is stem-loop agnostic. A stretch of nucleotide positions, which demonstrate high 149 probability of being paired across the entire dataset of RNAs, may form a functionally 150 important and evolutionarily conserved stem. Similarly, a batch of nucleotide positions with 151 low base pairing probabilities, which repeats itself across all RNAs in the dataset may form a 152 functionally important and evolutionarily conserved loop. Still, further analysis is necessary to 153 confirm both the stems and the loops. In all cases, within a PSR, the probability of each 154 nucleotide to be in a double-stranded conformation does not vary significantly across the 155 entire dataset of aligned RNAs and these positions are structure-conserved positions.

\section{Identification of Structured RNA Regions in H1N1 Influenza Virus mRNAs}

157 We applied RNA ISRAEU to predict evolutionarily conserved RNA structures of influenza A 158 virus (IAV) $(28,29)$.

159 We selected sequences encoded by the complete genomes of 107 pre-pandemic (1999 to 160 2009) and 173 pandemic (post-2009) human H1N1 strains (Supplementary Table 2). In 1612009 , a swine IAV strain was introduced into man and rapidly replaced the circulating 162 strains. All mRNAs selected for a given gene were of the same length. For each of the 10 
163 major viral mRNAs, we predicted structured regions and calculated sequence variation.

164 Profiles for non-pandemic and pandemic NS2 mRNA are depicted in Figure 2 (profiles for

165 other mRNAs are presented in Supplementary Figures 2-38).

166142 and 134 PSRs were identified in non-pandemic and pandemic influenza mRNAs

167 respectively. The length of PSRs varies from 5 to 121 nucleotides for non-pandemic and

168 from 5 to 103 nucleotides for pandemic strains (Table 1). The number of PSRs varies from 2

169 for NS2 to 31 for NP (Table 1). Only a fraction of PSRs overlap between pandemic and non-

170 pandemic strains. In some mRNAs (namely PB1, PB2, PA, HA and NA), the percentage of

171 such non-overlapping regions is higher than $70 \%$. The location of each PSR are presented

172 in Supplementary Table 3.

173 In comparing PSR profiles with the profiles of mean pairing probabilities, we found two

174 evolutionarily conserved structural elements. One is located between positions 105 and 132

175 in non-pandemic NS2 mRNA (Figure 2), which contains a previously unknown predicted

176 conserved hairpin (Figure 3). Nucleotides 105 to 114 and 123 to 132 have a strong predicted

177 tendency to be paired while intervening nucleotides 115 to 124 have a strong tendency to be

178 unpaired. By comparing Figures 2(b) and 2(e), one can predict that this new hairpin structure

179 also exists in pandemic NS2 influenza mRNAs. The second novel PSR identified in non-

180 pandemic NS2 mRNA is between positions 24 and 89 (Figures 2 and 3). In this case

181 pandemic mRNAs contain only the PSR created by nucleotides 40 to 73 .

\section{Oligonucleotides complementing the stem of the newly Predicted Structured Regions} 183 interfere with in vitro viral replication

184 To test the computationally predicted RNA structured regions, we have designed

185 oligonucleotides complementing the stem and the loop, as well as two controls of the same

186 length. The first control did not complement any sequence within the viral or human genome

187 while the other control bound a non-structured region adjacent to the PSR. The MDCK cell

188 monolayer was either transfected with one of the oligonucleotides and then infected with

$189 \mathrm{~A} / \mathrm{California} / 7 / 09$ strain or the cells were infected without prior transfection. The transfection

190 doze was not toxic for the cells as it was proven by the cell viability assay. Twenty four hours

191 post transfection and infection, the viral replication was assessed by developing the cell

192 monolayer with anti-NP ELISA. We observed that only the decamer complementing the stem

193 of the computationally predicted hairpin has significantly reduced the viral replication

194 comparing to the controls. Neither the oligonucleotide complementing the loop, nor the two

195 control oligos had a statistically significant effect on the in vitro viral replication (Figure 4).

\section{Location of the Most Mutable Positions}

197 We distinguish between two types of positions in the influenza genome - the mutable 198 positions which mutate quite frequently, and conserved positions. We tested if positions 199 mutating more often cluster outside of PSRs, while conserved positions are predominantly 200 located within the PSRs. The numbers for both types of positions in every mRNA of 201 pandemic and non-pandemic H1N1 strain are provided in the Supplementary Table 1. 202 Percentage of highly mutable positions in influenza mRNAs varies in a range from $7.9 \%$ in $203 \mathrm{M} 1$ to $15.5 \%$ in NA and from $6.8 \%$ in M2 to $15.8 \%$ in HA for non-pandemic and pandemic 204 influenza strains respectively. Among the highly mutable positions from $56.1 \%$ for NS1 to 
$20588.4 \%$ for NP and from $50.0 \%$ for M2 to $85.1 \%$ for $\mathrm{M} 1$ are third-codon positions for non206 pandemic and pandemic influenza mRNAs respectively. Results presented at plots (c) and 207 (f) of Figure 2 and Supplementary Figures 2-38 demonstrate that the most mutable positions 208 are randomly distributed within each mRNA and do not form clusters. Absence of 209 relationship between mutability value for every nucleotide position and corresponding value 210 of moving average of individual standard deviations of the probabilities of nucleotides to be 211 paired was confirmed by calculating Pearson correlation coefficients (Supplementary Table 4 212 and Supplementary Figures 39-58). All correlation coefficients were in a range from 0.006 to 213 0.222. This result refutes the intuitive notion that location of mutable positions would 214 correspond to the least structured RNA regions, while sequence conserved positions would 215 be collocated with the most structured RNA regions.

\section{Comparison of Mutation's Effect on RNA PSRs}

217 We generated two groups of in silico mutants by introducing synonymous mutations into 218 influenza mRNAs. In the first group, mutations were introduced only into positions that are 219 highly prone to being mutated; in the second one, mutations were introduced only into 220 sequence conserved positions. The number of introduced in silico mutations was 221 proportional to the length of every mRNA (Table 1). The effects of two groups of in silico 222 mutations on structured RNA regions were compared, as described in the Materials and 223 Methods section (Table 2). The results of statistical tests (Table 2) demonstrate that for 224 majority of mRNAs the mutations introduced into sequence conserved positions have a 225 greater effect on PSRs than mutations introduced into the mutable positions. This result 226 stands out the most in mRNAs of non-pandemic NP, M2, and NS1 genes and pandemic 227 NS2 gene.

\section{DISCUSSION}

230 Evolutionarily conserved RNA structural elements may perform important biological 231 functions. Hence, identification and/or prediction of such elements can help in the 232 understanding of the mechanism of RNA functions. This is true for identification of not only 233 paired regions (stems), but loops too. In fact, kissing loop interactions are a common type of 234 tertiary interaction motif in RNA that brings terminal loops together through Watson-Crick 235 base pairing. Also, bulged nucleotides in the loop-loop interaction can be critical for ligand236 dependent regulation. Yet, despite many efforts, it has still been a challenge to introduce an 237 objective, quantitative, biologically meaningful and computationally friendly definition of what 238 a "structured" RNA region is. Therefore, we had to propose a new definition and a new 239 computational methodology free of these shortcomings.

240 In analyzing an individual RNA sequence, one has little chance to distinguish a biologically 241 important structure formed by a folded molecule from simply a random shape with no 242 biological importance. However, if one observes the same RNA configuration conserved and 243 repeated across all related RNA sequences isolated from different strains and/or species, 244 this increases the likelihood of biologically significant RNA structure. Following this logic, a 245 definition of a structured RNA region should be based on a dataset of multiple aligned RNA 246 sequences. Thus, assume that some structural element in a particular location is of such 
247 importance that it is present across all the strains. In this case, nucleotides in positions 248 correspondent to the stem would have very high base pairing probabilities in all aligned RNA 249 sequences of the dataset, while nucleotides in positions correspondent to the loop would 250 have very low base pairing probabilities in all the strains. At the same time, nucleotides 251 correspondent to a potential bistable structure would have their base pairing probabilities neither too high nor very low across all RNA sequences. Therefore, we propose that "structured RNA regions" are defined as the patterns of probability values of the nucleotides to be paired, which are manifested across the spectrum of strains and/or organisms. This definition equally considers the conservation of stems, loops and potential bistable structures while also providing a computationally friendly quantitative definition for the degree of RNA structure conservation.

258 Mathematically, the fact that nucleotides in a particular position in each RNA of a dataset are 259 likely to belong to an evolutionarily conserved structural element means that if we collect 260 values of pairing probabilities for this nucleotide from each RNA sequence in the dataset, 261 and build a sample of these values to calculate its standard deviation, this standard deviation 262 will be relatively low compared with the majority of standard deviations for other positions. 263 Indeed, if this standard deviation is low, it means that mutations occurring in the analyzed 264 RNA do not affect the base pairing probability of a nucleotide in this position across the 265 spectrum of strains. Thus, it is most likely that mutations affecting pairing probability for this 266 nucleotide are filtered out. This is a good indicator of evolutionary conservation and the 267 biological importance of the RNA structure in this position. In contrary, if the standard 268 deviation is high, it means that the correspondent nucleotide is very likely to be bonded in some strains but not in others; hence, the presence of any crucial RNA structure at this position is unlikely (unless there is a bistable secondary structure in this area playing roles in different functions). If an RNA contained five consecutive nucleotides with low standard deviations of their mean pairing probabilities, the region was considered structured.

\section{Applications of a Newly Introduced Computational Definition}

274 Introduction of a new definition adequately describing the subject matter under study and development of a new technique for analysis, however, are only as good as they can be applied to a multitude of biological phenomena, generate new observations and experimentally testable hypotheses, explain old conundrums, and generate new questions (41). The presented approach was used to examine the existence of structured RNA regions in mRNAs of pandemic and non-pandemic influenza A H1N1 virus. This method revealed that influenza mRNAs contain nucleotide positions highly conserved in their base pairing 281 probabilities. For every analyzed RNA type, such positions group together and constitute well-defined structured RNA regions, while the rest of the RNA molecule is significantly less structured. To the best of our knowledge, such mosaic structurization of RNA molecules was not reported previously. In vitro testing has confirmed that interfering with a stem of a previously unknown computationally predicted RNA structured region indeed reduces viral replication. We expect that future experimental testing will reveal the functions, these evolutionarily conserved RNA secondary structures, perform during the course of viral infection. 
289 We hypothesized that mosaic structurization of influenza mRNAs may explain a long-

290 standing conundrum of why different nucleotides in influenza genome mutate with such a

291 varied frequency. The enormous influence of amino acid conservation could explain only a

292 part of this phenomenon because many nucleotide substitutions are synonymous ones thus

293 cannot be explained by amino acid conservation. The first hypothesis was that if a mutation

294 happens within a structured RNA region it would disrupt the structure and be filtered out.

295 Thus, even if a mutation rate was the same for all nucleotides, the only mutations observed

296 in nature would be those happening outside of the structured RNA regions (PSR) and

297 neutral for RNA structures. If an exact picture of each RNA structure was available, it would

298 be possible to define structurally disruptive mutations visually as those that change the

299 shape(s) of the structure(s). However, modern computational methods do not make it

300 possible to predict exact RNA structures for long RNA molecules. Such predictions are

301 inaccurate and cannot be relied upon (14, 42-44). Thus, we had to define structurally

302 disruptive mutations based on the number of nucleotides in structured RNA regions, which

303 would change their W-C pairing probabilities to a level aberrant of their naturally observed

304 range. Contrary to original expectations, we showed that the nucleotide positions which are

305 the least prone to being mutated do not collocate with regions of conserved RNA structures.

306 Instead, the frequently and/or rarely mutating positions are randomly spread along the RNA

307 sequences. Although it was demonstrated that the most frequently mutating positions within

308 influenza genome are not collocated within unstructured RNA regions, this finding does not

309 refute the main hypothesis that states: "Mutations, which occur in nucleotide positions that

310 are the most prone to single nucleotide polymorphisms, have less of an effect on structured

311 RNA regions than mutations, which occur in positions that are less likely to be changed".

312 A mutation does not necessarily have to take place inside the PSR in order to be disruptive

313 for a structure. For example, prior to mutation, a particular $\mathrm{G}$ was paired to a particular $\mathrm{C}$

314 forming a structure. If a mutation outside the structure changes some $A$ to $C$, it may become

315 a new paring partner for the $G$, thereby leading to an energetically more favorable RNA

316 folding and disrupting the original structural element. This effect may be especially strong if

317 mutations outside of the PSRs occur in combinations. Also, mutations in certain positions

318 may have a greater effect on RNA structures than that of other positions. Thus, if RNA

319 structures should indeed remain intact for successful viral propagation, all positions, SNPs in

320 which would have a striking effect on the structures, would seem as rarely mutating

321 compared to those positions, SNPs in which would have little effect on the structures. The

322 results presented here support this hypothesis. We demonstrated for some influenza

323 mRNAs that in silico mutations introduced into nucleotide positions, which mutate in the wild

324 less frequently, would possess a greater disruptive effect on areas of conserved RNA

325 structures than in silico mutations in positions which are known to mutate more frequently.

326 As a result, mutations deleterious for vital RNA structures would be eliminated due to the

327 negative selection pressure. This demonstrates that conservation of RNA structures could

328 be a contributing mechanism defining a highly differential mutation rate for different influenza

329 nucleotide positions. Additionally, the computational conclusion stipulates a direction for

330 experimental testing. Although it is time/cost-consuming, it is possible to test RNA shapes

331 experimentally $(4,45-49)$. If our hypothesis is correct, then influenza mRNAs observed in

332 nature and those RNAs carrying mutations, which we predicted to be structurally non-

333 disruptive, would possess similar RNA structures. By contrast, introducing into the RNA 
334 sequence mutations, which are predicted to disrupt structured RNA regions, would eliminate

335 at least some of the RNA structures vital for a virus.

\section{Identification of Evolutionarily Conserved RNA Structural Elements}

337 Plotting a graph with nucleotide positions on axis $X$ and standard deviations of nucleotide 338 paring probabilities for these positions on axis $Y$ shows stretches along the RNA sequence 339 with low standard deviations. These areas potentially have conserved RNA secondary 340 structural elements. However, these graphs alone do not demonstrate whether the 341 probability of a nucleotide to form bonds is high across different strains or low. In other 342 words, a structurization profile may help identifying localization of RNA PSRs, but it does not 343 indicate what kind of structure is there. Nevertheless, some assumptions about the RNA 344 shape can be made if we complement structurization profiles with profiles presenting mean 345 pairing probability for each nucleotide (i.e. for each nucleotide position in the RNA sequence, 346 the pairing probability values from every RNA in the dataset would be used to calculate the 347 mean for the position).

348 Extracting complex structures from comparing structurization profiles with profiles of mean pairing probabilities may require special analytical tools that are not a part of this first-stage 350 study. However, discovering the simplest hairpin structure may not require additional 351 instruments. Thus, when 10 nucleotides were found to possess very high means of 352 probabilities to be bonded in the entire dataset, followed by 8 structurally conserved 353 nucleotides which were apparently uncoupled, and then another 10 nucleotides that are 354 likely to be paired and complementing the first 10 as $\mathrm{W}-\mathrm{C}$ bonding partners, these findings 355 showed existence of a previously unknown evolutionarily conserved RNA hairpin structure. 356 In vitro testing has confirmed that interfering with a stem of a previously unknown 357 computationally predicted RNA structured region indeed reduces viral replication. We expect 358 that future experimental testing will reveal the functions, these evolutionarily conserved RNA 359 secondary structures, perform during the course of viral infection.

360 It would be important to test whether pandemic and seasonal influenza strains indeed share 361 some PSRs and whether the difference in RNA structurization may play a role in pandemic 362 vs. non-pandemic viral phenotypes. Another direction of the future research would be to 363 expand our computational definition of a structured RNA region to predict evolutionarily 364 conserved RNA tertiary structures, especially in those RNAs that are hard to study by high365 resolution experimental methods (50). In addition to helical segments, RNAs can fold into 366 complex three-dimensional shapes. Computational modeling of RNA tertiary structures and 367 determining of three-dimensional shapes of complex RNAs constitutes a major intellectual 368 challenge (51-55). Thus, the most practical way to expand the proposed computational 369 method to studying RNA 3D structures would be to incorporate RNA 3D structural modules 370 that define sets of non-Watson-Crick base pairs embedded in WC pairs $(56,57)$.

371 Novel Approach for the Rational Design of Live-Attenuated Vaccines and Anti-Viral 372 Therapies

373 The method we proposed and applied to define structured RNA regions revealed several 374 areas possessing conserved secondary structures in mRNAs of H1N1 influenza virus. As a 375 next step, these structures have to be confirmed by in vitro analysis and their biological roles 
376 have to be assessed in vitro and/or in vivo. Potentially, structurally conserved RNA regions

377 of viral RNAs may become a novel class of anti-viral drug targets. For example, anti-viral

378 agents selectively disrupting RNA structures vital for a viral life cycle may become a new

379 class of anti-viral therapies. As a preliminary proof of concept, we have demonstrated that an

380 oligonucleotide binding the computationally predicted stem of a hairpin in a PSR, indeed acts

381 as an anti-viral agent reducing in vitro viral replication. In contrast, statistically significant

382 effect on viral replication was not observed if the infected cells transfected with the oligos of

383 the same length, which bind outside of the predicted hairpin or do not bind to anything at all.

384 Interestingly, even an oligonucleotide complementing the loop of this hairpin was unable to

385 reduce viral replication in a statistically significant manner. Thus, the anti-viral effect was

386 specific to disrupting the hairpin's stem. RNA ISRAEU allows rapid rational design of

387 oligonucleotide cocktails interfering with multiple computationally predicted structures, so no

388 single or few mutations would result in a resistant viral strain.

389 Several approaches have been proposed for analysis of impact of SNPs on RNA structures 390 and deleterious mutation prediction (1, 58), including RNAsnp (59, 60), SNPfold (61), 391 RNAmute $(62,63)$, RNAmutants (64), and RDMAS (65). However, all these methods 392 compare structures of the original and mutated RNAs assessing the distance, the effect on 393 the RNA structure caused by SNPs. Although these methods are productive for the tasks 394 they were developed for, they cannot be applied to our problem. We do not compare 395 structures of an original and an altered RNA sequences. Instead, we compare structures of 396 hundreds of RNA sequences without attributing any of them the "original" status. Therefore, 397 the approach proposed here allows us to define: (i) a naturally occurring range of 398 probabilities, which represents a range of probability values that are the most likely to be 399 observed for natural RNA strains (see Quantitative Assessment of Mutation's Effect on RNA 400 PSRs in the Materials and Methods section for the specification) for every nucleotide 401 position within an RNA region possessing an evolutionarily conserved structure; (ii) 402 mutation(s) that would change base pairing probabilities within the structured RNA regions to 403 an extent that the new probabilities would not belong to a naturally occurring range for 404 corresponding positions.

405

406 Finally, we propose a new approach for the rational design of attenuated vaccines that would 407 be based on predicting mutations disruptive for conserved RNA structures and introducing 408 such mutations into viral genome. Indeed, disruption of an mRNA structure may serve as a 409 functional gene knock out reducing expression of a viral gene to a level insufficient for viral 410 cycle (12). Viral strain possessing such RNA can be administered to induce an immune 411 response with little risk for a patient. Such attenuated viral strains can be grown on 412 supporting cell lines actively expressing the limiting protein. Although LAVs are the most 413 successful achievements in the history of public health (38), we believe there were no prior 414 attempts to create LAVs based on perturbation of RNA structures. 
419 Selecting H1N1 influenza mRNAs for this work constituted a crucial initial step. Influenza A

420 genome consists of eight segments encoding seventeen proteins (66). Seven of those

421 proteins were excluded from the analysis due to the limited information about them. It is

422 known, however, that different influenza segments have different mutation rates (67). To

423 eliminate potential bias that can be caused by disproportional representation of similar

424 hemagglutinin (HA) and neuraminidase (NA) sequences (these two influenza genes are

425 sequenced more often than the others because they constitute major viral antigens) and to

426 compare evolutionary structure conservation between different influenza mRNAs, only

427 completely sequenced influenza genomes were utilized in the analysis. An influenza genome

428 was considered completely sequenced if it had no missing parts, no unknown nucleotides,

429 and if sequences of the ten major mRNAs (namely, PB1, PB2, PA, HA, NP, NA, M1, M2,

430 NS1, and NS2) were known. In order to further increase coherence of the dataset, only

431 human influenza strains were utilized; other hosts were excluded because they demonstrate

432 different characteristics (68). Finally, only those strains possessing the identical length of

433 each influenza mRNA were selected. The fact that every mRNA of the same type has the

434 same length in every viral genome selected eliminates potential mistakes, which could be

435 introduced by effects of deletion and insertion polymorphisms (DIPs) on RNA secondary

436 structures. Sequences of pandemic and non-pandemic complete influenza genomes

437 satisfying the above mentioned criteria were downloaded from the Influenza Virus Resource

438 (http://www.ncbi.nlm.nih.gov/genomes/FLU/FLU.html) (69).

\section{Filtering Redundant Sequences}

440 Redundancy of data may introduce significant bias. To avoid it, one must only use a 441 representative subset of sequences instead of analyzing all possible strains. Therefore, 442 strains that were too similar were eliminated from further analysis; and, a non-redundant 443 subset of strains was created. Any two strains in the non-redundant subset possess no less 444 than 50 nucleotide differences per complete genome. In short, the first strain was chosen 445 randomly from the dataset described in the previous section, then added to the non446 redundant subset. Then, a different strain was randomly chosen and added to the non447 redundant subset only if the newly chosen sequence had at least 50 nucleotide differences 448 versus all strains in the non-redundant subset. This step was repeated until no more 449 sequences could be added to the non-redundant subset. The described procedure was done 450 separately for the pandemic and non-pandemic influenza datasets described above.

\section{Structural Conservation of a Nucleotide Position}

452 As a first step, for each mRNA sequence in the datasets, the probability of every nucleotide 453 within an RNA chain to be coupled via W-C bond was calculated. For that purpose, the 454 RNAfold tool from the Vienna RNA package was used (70). RNAfold was used with the 455 command line options $-p$ that calculates the partition function and base pairing probability 456 matrix, --noLP that disallows base pairs that can only occur as helices of length 1 , and the 457 default folding temperature fixed at $37^{\circ} \mathrm{C}$. As a result, if a non-redundant dataset consisted 458 of $\mathrm{N}$ sequences, a sample of $\mathrm{N}$ probability values would be created for each position (exactly $459 \mathrm{~N}$ for a position, in which there is no deletion/insertion polymorphisms) within analyzed RNA. 460 The standard deviation was calculated for every sample. The procedure described above 461 was conducted for each of ten mRNAs from both subsets. Thus, we have calculated 
462 standard deviations of the probabilities of nucleotides to be paired for every nucleotide

463 position within each of ten influenza mRNAs in both pandemic and non-pandemic datasets.

464 To smooth stochastic fluctuations, moving averages of individual standard deviations with

465 the sliding window of 5-nt length were calculated (Figure 2). To determine structure

466 conserved positions, all moving average values of individual standard deviations from all

467 mRNAs were combined to one dataset of moving averages, and the mean value and

468 standard deviation of the values in that dataset were calculated. If an individual moving

469 average calculated for a particular position was smaller than the overall mean of moving

470 averages minus the overall standard deviation of moving averages, the correspondent

471 position was considered "structure-conserved".

\section{RNA Structurization and Structured RNA Regions}

473 As described above, noticeable areas of structure-conserved positions possessing low 474 standard deviation values were observed. Areas possessing at least five consequent 475 structure-conserved nucleotides were defined as "structured RNA regions". The described 476 procedure was repeated separately for pandemic and non-pandemic influenza strains.

\section{Mutability}

478 Intuitively, "mutability" demonstrates how likely it is for a nucleotide in a particular position to 479 be mutated. Mathematically, this simple notion is defined as the value of Shannon entropy 480 (35), which is calculated based on the frequencies of every ribonucleotide recorded in a 481 particular position, with a pseudocount regularizers equal to 1 being added to the frequency 482 of each of four ribonucleotides according to Laplace's rule. To identify nucleotide positions 483 that are the most/least prone to being mutated, the mutability value was computed for each 484 nucleotide position. The more variable a set of ribonucleotides observed in a particular 485 position, the higher the entropy. Then, all mutability values from all mRNAs were combined 486 into one dataset. Those positions that had their mutability values higher than the 80th 487 percentile of the dataset were considered as mutable positions. In contrast, those positions 488 that did not contain SNPs among the sequences in the dataset were considered conserved 489 positions. The described procedure was repeated separately for pandemic and non490 pandemic influenza strains.

\section{Quantitative Assessment of Mutation's Effect on RNA PSRs}

492 Following the analysis discussed above, a new method was proposed and implemented, 493 which defines "structurally disruptive mutations" based on their effect on structured RNA 494 regions (PSRs). As described previously, two datasets of aligned influenza sequences were 495 created. For each individual RNA sequence within the datasets, the probability of each 496 nucleotide to be paired was computed. For every nucleotide position within coding regions of 497 influenza mRNA sequences, the mean value and the standard deviation of the probabilities 498 of nucleotides to be paired were calculated. Based on these values, a naturally occurring 499 range of probabilities was calculated for every nucleotide position within a PSR. A naturally 500 occurring range of probabilities was defined as a range of probabilities from the mean value 501 decreased by two standard deviations to the mean value increased by two standard 502 deviations. 
503 Apparently, a mutation occurring in an RNA sequence may change probability of forming

504 Watson-Crick pairs for multiple nucleotides within a particular sequence. For some of those

505 nucleotides, their new probability values would still belong to the naturally occurring range of

506 probabilities for this position. For other positions, the mutation would change their pairing

507 probabilities to an extent that the new probabilities would not belong to their naturally

508 occurring range. A quantitative effect of mutation(s) on RNA structurization is defined as a

509 number of nucleotides within structured RNA regions (PSRs), which would change their

510 probabilities to an extent that the new probabilities would not belong to a naturally occurring

511 range for corresponding positions.

\section{Statistical Analysis: Do Mutations Taking Place in the Most vs. Least Often Mutating}

513 Positions Have a Different Effect on RNA Structurization?

514 Some positions in influenza genome are more prone to being mutated than others. The 515 ability to define quantitatively effects of mutations on RNA structurization permitted the 516 opportunity to propose a method for assessment, if mutations taking place in the frequently 517 mutating positions have the same effect on RNA structurization as mutations occurring in the 518 conserved ones. Two sets of in silico mutants were generated introducing synonymous 519 mutations in nucleotide positions that are either the most or the least prone to being 520 mutated. These two sets of mutations were compared for their effect on structured RNA 521 regions.

522 In order to normalize for the length difference among influenza mRNAs, the number of 523 changed nucleotides, which were introduced into each mRNA, was in proportion to the 524 length of the mRNA (Table 1). The required number of synonymous SNPs was introduced 525 into every mRNA sequence from the original datasets. In order to generate an in silico 526 mutant from an original mRNA sequence, the required number of positions that are the most or the least prone to being mutated were randomly selected. Every codon, which contains the selected position, was changed to an alternative one encoding the same amino acid with the condition that the new codon is not observed in the particular position in any mRNA sequence from the datasets. Influenza mRNAs contain relatively high number of conserved positions and relatively few often mutating ones. As a result, for every mRNA, the number of in silico mutants with SNPs in conserved positions was equal to the number of wild type influenza strains in the datasets. However, due to a small number of frequently mutating positions, it was impossible for some mRNAs to generate the same number of unique mutants by introducing SNPs only to positions prone to being mutated. In those cases, all 536 possible mutants were kept for further analysis - namely, 103 for non-pandemic M2, 95 for 537 pandemic M2, and 109 for pandemic NS2.

538 For each computer-generated mutant, the probability of every nucleotide to be in a double539 stranded conformation was calculated. Based on those probabilities, we calculated the 540 number of nucleotides within structured RNA regions (PSRs), which changed their 541 probability of being paired to a value outside of the naturally occurring range of probabilities 542 for this position. Such numbers were combined into two datasets: one for mutations 543 introduced into highly mutable positions and another - for mutations introduced in highly 544 conserved positions. The Mann-Whitney $U$ test was conducted for comparing these two 545 datasets. The significance level for the test was Bonferroni-corrected by dividing the 
546 significance level of $5 \%$ by the total number of mRNAs in influenza virus, i.e. 10. The

547 described procedure was repeated separately for pandemic and non-pandemic influenza

548 strains.

549 Profiles of Mean Pairing Probabilities

550 Profiles of mean pairing probabilities were created for influenza mRNAs (Figure 2 and 551 Supplementary Figures 2-38). These profiles demonstrate how likely on average each 552 nucleotide within an mRNA is to be paired based on an analysis of the entire dataset of 553 sequences. As mentioned, for every RNA sequence in the dataset, the probability of every 554 nucleotide within the RNA chain to be coupled via W-C bond was calculated. Then, for every 555 nucleotide position, we computed the mean for probability values of this nucleotide based on 556 all RNA sequences. The resulting series of means is used as a profile of mean pairing 557 probabilities for a particular mRNA. The same work was performed for every influenza 558 mRNA.

\section{Virus and Cells}

560 Influenza virus A/California/7/09 ( $\mathrm{H} 1 \mathrm{~N} 1 \mathrm{pdm})$ was provided by the Research Institute of 561 Influenza museum of viruses, Saint-Petersburg, Russian Federation. The $50 \%$ tissue culture 562 infective dose (TCID50) of this virus strain was defined by Reed-Muench method (72). The 563 aliquots of virus were stored at $-80 \mathrm{oC}$. According to the American Tissue Madin-Darby 564 canine kidney (MDCK) cell culture was provided from the cell collection of Research Institute 565 of Influenza, Saint-Petersburg, Russian Federation. Cells were cultivated in cultural flasks 566 using minimum essential medium Eagle alpha modification (aMEM, Biolot) with 2mM L567 glutamine supplemented with $10 \%$ heat-inactivated fetal bovine serum (FBS, GIBCO, USA).

\section{Design of Antisense DNA-oligonucleotides}

569 We designed antisense oligonucleotides, which may potentially disrupt the aforementioned 570 predicted RNA-structure. A random oligonucleotide, "rand10", with minimal probability of 571 having targets in the human hosts and viral genome was used as a control . Another control, 572 "off10", was an oligonucleotide with a target to the adjacent region on the NS2 gene mRNA 573 (Table 3).

\section{Cell Viability Assay}

576 The cell viability was determined 24 hours post infection and transfection by 577 microtetrazolium test (MTT assay). A solution of MTT [3-(4,5-dimethylthiazol-2-yl)-2,5578 diphenyltetrazolium bromide] (Sigma) at a concentration of $2,5 \mathrm{mg} / \mathrm{ml}$ was prepared in PBS. 579 The medium was removed, the cells were washed once with PBS, MTT solution was added 580 into the wells (100 ul/well). The cells were incubated at $37^{\circ} \mathrm{C}$ and $5 \% \mathrm{CO} 2$ for 4 hours and 581 then the solution was removed and $96 \%$ ethanol (100 ul/well) was added for formazan 582 crystals dissolving. The absorbance signal was measured using multifunctional reader 583 CLARIOstar ${ }^{\circledR}$ (BMG LABTECH, Germany) at $535 \mathrm{~nm}$.

\section{Virus Infection and Transfection}


585 The cells were detached by $0,25 \%$ trypsin/EDTA solution (Invitrogen) for $5 \mathrm{~min}$ and plated in 586 96-well plates (Nucl) at 104 cells per well the day before the infection experiment. Cells were 587 washed twice with Dulbecco's Phosphate-Buffered Saline (DPBS, GIBCO) and infected with $588 \mathrm{~A} /$ California/7/09 (H1N1pdm) viral strain in 100TCID50 dose per well. The medium for cells 589 infection was minimum essential medium Eagle alpha modification (aMEM, Biolot) with 2mM 590 L-glutamine, $2,5 \mathrm{ug} / \mathrm{ml}$ trypsin TPCK-treated from bovine pancreas (TPCK, Sigma) and $591 \quad 1: 100$ antibiotic-antimycotic (100X, GIBCO). Inoculation was conducted at $37^{\circ} \mathrm{C}$ and $5 \%$ $592 \mathrm{CO} 2$ for 60 minutes. Then the medium was removed and the cells were transfected using $593100 \mu \mathrm{l}$ of OptiPro SFM medium (GIBCO) contained $10 \mu \mathrm{M}$ of DNA-oligonucleotides and 0.7 $594 \mu \mathrm{l} /$ well of Lipofectamine 2000 (Invitrogen) according to the manufacturer's protocol. In 595 addition, the transfection medium is also supplemented with 1:100 antibiotic-antimycotic $596(100 \mathrm{X})$ and 2,5 ug/ml TPCK. Viral control samples were also transfected with lipofectamin 2000 only, without any oligonucleotides. Four hours post-transfection, the medium was replaced with fresh aMEM (Biolot) which contained $2 \mathrm{mM} \mathrm{L-glutamine,} \mathrm{2,5} \mathrm{ug/ml} \mathrm{TPCK} \mathrm{and}$ 599 1:100 antibiotic-antimycotic (100X). Twenty four hours post- infection, cells were used for the 600 further relative ELISA analyses. Each treatment was performed in triplicates.

\section{Enzyme-Linked Immunosorbent Assays (ELISA)}

602 Twenty four hours post influenza virus infection and transfection with oligonucleotides, 603 continuity of the cell monolayer was assessed microscopically. Then, medium was removed 604 and the MDCK cells in 96-wells Nunc plates were fixed with $150 \mu \mathrm{l}$ per well of cold $80 \%$ 605 acetone at $4^{\circ} \mathrm{C}$ for 30 minutes. The fixed samples were washed three times with phosphate 606 buffered saline containing $0.05 \%$ Tween (PBS-T) and blocked with $5 \%$ milk dissolved in 607 PBST $\left(200 \mathrm{ul} /\right.$ well) for 30 minutes at $37^{\circ} \mathrm{C}$. The fixed cells were incubated with $1 \mathrm{ug} / \mathrm{ml}$ 608 mouse antibody against NP-protein (100 ul/well) produced in the Influenza Research 609 Institute (clone $4 \mathrm{H} 1$ ) at $37^{\circ} \mathrm{C}$ for 1 hour. After the next three washes the secondary goat anti610 mouse antibody conjugated with horseradish peroxidase (GAM-HRP, BioRad, USA) was 611 added at $1 \mathrm{ug} / \mathrm{ml}\left(100 \mathrm{ul} /\right.$ well) and incubated for 1 hour at $37^{\circ} \mathrm{C}$. Cells were washed three 612 times with PBS-T followed by adding TMB Peroxidase EIA Substrate Kit (Bio-Rad, USA) 613 according to manufacturer's instructions for further absorbance analysis. The absorbance 614 was measured using multifunctional reader CLARIOstar ® (BMG LABTECH) as delta optical 615 density OD 450 - OD 655. The absorbance signal from uninfected cells was taken as zero 616 and was subtracted from the obtained values of the samples. The results were presented 617 relative to infection control.

\section{Statistical Analysis of Viral Replication Inhibition Assay}

619 Data shown are means +/- SD as percentage of untreated "Flu" group. P-values for 620 comparing the four treatment groups with the untreated group (Flu) were calculated using 621 student $\mathrm{T}$ test. The significance level for the test was Bonferroni-corrected by dividing the 622 significance level of 0.05 by the total number of group comparisons, i.e. 10. Analysis was 623 performed using the $\mathrm{R}$ software. 
626 This work was supported by CureLab Oncology, Inc.; and the Deutsche 627 Forschungsgemeinschaft International Research Training Group 'Regulation and Evolution

628 of Cellular Systems' [GRK 1563]. NF and JWY were supported by the Division of Intamural 629 Research, NIAID, Bethesda MD, USA.

\section{ACKNOWLEDGEMENTS}

632 The authors would like to thank Prof. Dmitrij Frishman, a Ph.D. advisor of Andrey Chursov. 633 This work was partially performed during AC term in Dmitrij Frishman's lab. The authors 634 would also like to thank Prof. Adolfo Garcia-Sastre for illuminating comments on the article 635 and the methodology, and Prof. Andrey Mironov and Prof. Ilya Muchnik for intellectually 636 stimulating discussions.

\section{7}

\section{REFERENCES}

639 1. Bevilacqua PC, Blose JM. Structures, kinetics, thermodynamics, and biological functions of 640 RNA hairpins. Annu Rev Phys Chem. 2008;59:79-103.

641 2. Chursov A, Frishman D, Shneider A. Conservation of mRNA secondary structures may filter 642 out mutations in Escherichia coli evolution. Nucleic Acids Res. 2013;41(16):7854-60.

643 3. Wan Y, Kertesz M, Spitale RC, Segal E, Chang HY. Understanding the transcriptome through 644 RNA structure. Nat Rev Genet. 2011;12(9):641-55.

645 4. Wan Y, Qu K, Ouyang Z, Kertesz M, Li J, Tibshirani R, et al. Genome-wide measurement of 646 RNA folding energies. Mol Cell. 2012;48(2):169-81.

647 5. Dethoff EA, Chugh J, Mustoe AM, Al-Hashimi HM. Functional complexity and regulation 648 through RNA dynamics. Nature. 2012;482(7385):322-30.

649 6. Geisler S, Coller J. RNA in unexpected places: long non-coding RNA functions in diverse 650 cellular contexts. Nat Rev Mol Cell Biol. 2013;14(11):699-712.

651 7. Rouskin S, Zubradt M, Washietl S, Kellis M, Weissman JS. Genome-wide probing of RNA 652 structure reveals active unfolding of mRNA structures in vivo. Nature. 2014;505(7485):701-5.

653 8. Shabalina SA, Spiridonov NA, Kashina A. Sounds of silence: synonymous nucleotides as a key 654 to biological regulation and complexity. Nucleic Acids Res. 2013;41(4):2073-94.

6559 9. Mortimer SA, Kidwell MA, Doudna JA. Insights into RNA structure and function from 656 genome-wide studies. Nat Rev Genet. 2014;15(7):469-79.

657 10. Tuplin A. Diverse roles and interactions of RNA structures during the replication of positive658 stranded RNA viruses of humans and animals. J Gen Virol. 2015;96(Pt 7):1497-503.

659 11. Chursov A, Kopetzky SJ, Bocharov G, Frishman D, Shneider A. RNAtips: Analysis of 660 temperature-induced changes of RNA secondary structure. Nucleic Acids Res. 2013;41(Web Server 661 issue):W486-91.

662 12. Ilyinskii PO, Schmidt T, Lukashev D, Meriin AB, Thoidis G, Frishman D, et al. Importance of 663 mRNA secondary structural elements for the expression of influenza virus genes. OMICS.

664 2009;13(5):421-30.

66513.

666 Chursov A, Kopetzky SJ, Leshchiner I, Kondofersky I, Theis FJ, Frishman D, et al. Specific temperature-

667 induced perturbations of secondary mRNA structures are associated with the cold-adapted

668 temperature-sensitive phenotype of influenza A virus. RNA Biol. 2012;9(10):1266-74.

669 14. Seetin MG, Mathews DH. RNA structure prediction: an overview of methods. Methods Mol

670 Biol. 2012;905:99-122. 
671 15. Gruber AR, Findeiss S, Washietl S, Hofacker IL, Stadler PF. RNAz 2.0: improved noncoding

672 RNA detection. Pac Symp Biocomput. 2010:69-79.

673 16. Soldatov RA, Vinogradova SV, Mironov AA. RNASurface: fast and accurate detection of

674 locally optimal potentially structured RNA segments. Bioinformatics. 2014;30(4):457-63.

675 17. Washietl S, Pedersen JS, Korbel JO, Stocsits C, Gruber AR, Hackermuller J, et al. Structured

676 RNAs in the ENCODE selected regions of the human genome. Genome Res. 2007;17(6):852-64.

677 18. Hofacker IL, Fekete M, Stadler PF. Secondary structure prediction for aligned RNA

678 sequences. J Mol Biol. 2002;319(5):1059-66.

679 19. Knudsen B, Hein J. RNA secondary structure prediction using stochastic context-free

680 grammars and evolutionary history. Bioinformatics. 1999;15(6):446-54.

681 20. Knudsen B, Hein J. Pfold: RNA secondary structure prediction using stochastic context-free

682 grammars. Nucleic Acids Res. 2003;31(13):3423-8.

683 21. Washietl S, Hofacker IL. Consensus folding of aligned sequences as a new measure for the

684 detection of functional RNAs by comparative genomics. J Mol Biol. 2004;342(1):19-30.

685 22. Gardner PP, Giegerich R. A comprehensive comparison of comparative RNA structure

686 prediction approaches. BMC Bioinformatics. 2004;5:140.

687 23. Chen C, Zhang H, Broitman SL, Reiche M, Farrell I, Cooperman BS, et al. Dynamics of

688 translation by single ribosomes through mRNA secondary structures. Nat Struct Mol Biol.

689 2013;20(5):582-8.

690 24. Schultes EA, Bartel DP. One sequence, two ribozymes: implications for the emergence of

691 new ribozyme folds. Science. 2000;289(5478):448-52.

692 25. Hobartner C, Micura R. Bistable secondary structures of small RNAs and their structural

693 probing by comparative imino proton NMR spectroscopy. J Mol Biol. 2003;325(3):421-31.

694 26. Weeks KM. Advances in RNA structure analysis by chemical probing. Curr Opin Struct Biol.

695 2010;20(3):295-304.

696 27. Thirumalai D, Hyeon C. RNA and protein folding: common themes and variations.

697 Biochemistry. 2005;44(13):4957-70.

698 28. Fitch WM, Leiter JM, Li XQ, Palese P. Positive Darwinian evolution in human influenza A

699 viruses. Proc Natl Acad Sci U S A. 1991;88(10):4270-4.

700 29. Nelson MI, Simonsen L, Viboud C, Miller MA, Taylor J, George KS, et al. Stochastic processes

701

702

703

704

705

706

707

708

709

710

711 are key determinants of short-term evolution in influenza a virus. PLoS Pathog. 2006;2(12):e125.

30. Furuse $Y$, Suzuki A, Kamigaki T, Oshitani H. Evolution of the $M$ gene of the influenza A virus in different host species: large-scale sequence analysis. Virol J. 2009;6:67.

31. Gultyaev AP, Fouchier RA, Olsthoorn RC. Influenza virus RNA structure: unique and common features. Int Rev Immunol. 2010;29(6):533-56.

32. Carrillo-Santisteve P, Ciancio BC, Nicoll A, Lopalco PL. The importance of influenza

prevention for public health. Hum Vaccin Immunother. 2012;8(1):89-95.

33. de Jong JC, Claas EC, Osterhaus AD, Webster RG, Lim WL. A pandemic warning? Nature. 1997;389(6651):554.

34. Garten RJ, Davis CT, Russell CA, Shu B, Lindstrom S, Balish A, et al. Antigenic and genetic characteristics of swine-origin $2009 \mathrm{~A}(\mathrm{H} 1 \mathrm{~N} 1)$ influenza viruses circulating in humans. Science.

712 2009;325(5937):197-201.

713 35. Shannon CE, Weaver W. The mathematical theory of communication. Urbana,: University of 714 Illinois Press; 1949. v (i.e. vii), 117 p. p.

715 36. Cox NJ, Kitame F, Kendal AP, Maassab HF, Naeve C. Identification of sequence changes in the

716 cold-adapted, live attenuated influenza vaccine strain, A/Ann Arbor/6/60 (H2N2). Virology.

717 1988;167(2):554-67.

718 37. Klimov Al, Cox NJ, Yotov WV, Rocha E, Alexandrova GI, Kendal AP. Sequence changes in the

719 live attenuated, cold-adapted variants of influenza A/Leningrad/134/57 (H2N2) virus. Virology.

720 1992;186(2):795-7. 
721 38. Ilyinskii PO, Thoidis G, Shneider AM. Development of a vaccine against pandemic influenza viruses: current status and perspectives. Int Rev Immunol. 2008;27(6):392-426.

39. Simmonds P, Tuplin A, Evans DJ. Detection of genome-scale ordered RNA structure (GORS) in genomes of positive-stranded RNA viruses: Implications for virus evolution and host persistence. RNA. 2004;10(9):1337-51.

40. Aouacheria A, Navratil V, Lopez-Perez R, Gutierrez NC, Churkin A, Barash D, et al. In silico whole-genome screening for cancer-related single-nucleotide polymorphisms located in human mRNA untranslated regions. BMC Genomics. 2007;8:2.

41. Shneider AM. Four stages of a scientific discipline; four types of scientist. Trends Biochem Sci. 2009;34(5):217-23.

42. Shapiro BA, Yingling YG, Kasprzak W, Bindewald E. Bridging the gap in RNA structure prediction. Curr Opin Struct Biol. 2007;17(2):157-65.

43. Marti-Renom MA, Capriotti E. Computational RNA structure prediction. Current Bioinformatics. 2008;3(1):32-45.

44. Chursov A, Walter MC, Schmidt T, Mironov A, Shneider A, Frishman D. Sequence-structure relationships in yeast mRNAs. Nucleic Acids Res. 2012;40(3):956-62.

45. Deigan KE, Li TW, Mathews DH, Weeks KM. Accurate SHAPE-directed RNA structure determination. Proc Natl Acad Sci U S A. 2009;106(1):97-102.

46. Mortimer SA, Trapnell C, Aviran S, Pachter L, Lucks JB. SHAPE-Seq: High-Throughput RNA Structure Analysis. Curr Protoc Chem Biol. 2012;4(4):275-97.

47. Loughrey D, Watters KE, Settle AH, Lucks JB. SHAPE-Seq 2.0: systematic optimization and extension of high-throughput chemical probing of RNA secondary structure with next generation sequencing. Nucleic Acids Res. 2014;42(21).

48. Kertesz M, Wan Y, Mazor E, Rinn JL, Nutter RC, Chang HY, et al. Genome-wide measurement of RNA secondary structure in yeast. Nature. 2010;467(7311):103-7.

49. Ding Y, Tang Y, Kwok CK, Zhang Y, Bevilacqua PC, Assmann SM. In vivo genome-wide profiling of RNA secondary structure reveals novel regulatory features. Nature. 2014;505(7485):696-700.

50. Ding F, Lavender CA, Weeks KM, Dokholyan NV. Three-dimensional RNA structure refinement by hydroxyl radical probing. Nat Methods. 2012;9(6):603-8.

51. Cruz JA, Blanchet MF, Boniecki M, Bujnicki JM, Chen SJ, Cao S, et al. RNA-Puzzles: a CASP-like evaluation of RNA three-dimensional structure prediction. RNA. 2012;18(4):610-25.

52. Miao Z, Adamiak RW, Blanchet MF, Boniecki M, Bujnicki JM, Chen SJ, et al. RNA-Puzzles Round II: assessment of RNA structure prediction programs applied to three large RNA structures. RNA. 2015;21(6):1066-84.

53. Tinoco I, Jr., Bustamante C. How RNA folds. J Mol Biol. 1999;293(2):271-81.

54. Laing C, Schlick T. Computational approaches to 3D modeling of RNA. J Phys Condens Matter. 2010;22(28):283101.

55. Laing C, Schlick T. Computational approaches to RNA structure prediction, analysis, and design. Curr Opin Struct Biol. 2011;21(3):306-18.

56. Leontis NB, Westh of E. Geometric nomenclature and classification of RNA base pairs. RNA. 2001;7(4):499-512.

57. Theis C, Zirbel CL, Zu Siederdissen CH, Anthon C, Hofacker IL, Nielsen H, et al. RNA 3D Modules in Genome-Wide Predictions of RNA 2D Structure. PLoS One. 2015;10(10):e0139900.

58. Barash D, Churkin A. Mutational analysis in RNAs: comparing programs for RNA deleterious mutation prediction. Brief Bioinform. 2011;12(2):104-14.

59. Sabarinathan R, Tafer H, Seemann SE, Hofacker IL, Stadler PF, Gorodkin J. RNAsnp: efficient detection of local RNA secondary structure changes induced by SNPs. Hum Mutat. 2013;34(4):54656.

769 60. Sabarinathan R, Tafer H, Seemann SE, Hofacker IL, Stadler PF, Gorodkin J. The RNAsnp web 770 server: predicting SNP effects on local RNA secondary structure. Nucleic Acids Res. 2013;41(Web 771 Server issue):W475-9. 
772 61. Halvorsen M, Martin JS, Broadaway S, Laederach A. Disease-associated mutations that alter the RNA structural ensemble. PLoS Genet. 2010;6(8):e1001074.

774 62. Churkin A, Barash D. RNAmute: RNA secondary structure mutation analysis tool. BMC 775 Bioinformatics. 2006;7:221.

776 63. Churkin A, Gabdank I, Barash D. The RNAmute web server for the mutational analysis of RNA secondary structures. Nucleic Acids Res. 2011;39(Web Server issue):W92-9.

778 64. Waldispuhl J, Devadas S, Berger B, Clote P. RNAmutants: a web server to explore the 779 mutational landscape of RNA secondary structures. Nucleic Acids Res. 2009;37(Web Server 780 issue):W281-6.

781 65. Shu W, Bo X, Liu R, Zhao D, Zheng Z, Wang S. RDMAS: a web server for RNA deleterious 782 mutation analysis. BMC Bioinformatics. 2006;7:404.

783 66. Vasin AV, Temkina OA, Egorov VV, Klotchenko SA, Plotnikova MA, Kiselev Ol. Molecular 784 mechanisms enhancing the proteome of influenza A viruses: an overview of recently discovered 785 proteins. Virus Res. 2014;185:53-63.

786 67. Webster RG, Bean WJ, Gorman OT, Chambers TM, Kawaoka Y. Evolution and ecology of influenza A viruses. Microbiol Rev. 1992;56(1):152-79.

790

791

792

793

794 ViennaRNA Package 2.0. Algorithms Mol Biol. 2011;6:26.

68. Brower-Sinning R, Carter DM, Crevar CJ, Ghedin E, Ross TM, Benos PV. The role of RNA folding free energy in the evolution of the polymerase genes of the influenza A virus. Genome Biol. 2009;10(2):R18.

69. Bao Y, Bolotov P, Dernovoy D, Kiryutin B, Zaslavsky L, Tatusova T, et al. The influenza virus resource at the National Center for Biotechnology Information. J Virol. 2008;82(2):596-601.

795 71. Zuker M. Mfold web server for nucleic acid folding and hybridization prediction. Nucleic 796 Acids Res. 2003;31(13):3406-15.

797 72. Reed L, Muench H. A simple method of estimating fifty per cent endpoints. Am J Hyg. $798 \quad 1938 ; 27: 493-497$ 
801 Table 1: Numbers of identified structured RNA regions within influenza mRNAs.

\begin{tabular}{|c|c|c|c|c|c|c|c|c|c|c|c|c|c|}
\hline \multirow{3}{*}{$\begin{array}{l}\text { Gene } \\
\text { name }\end{array}$} & \multicolumn{6}{|c|}{ Non-pandemic } & \multicolumn{6}{|c|}{ Pandemic } & \multirow{3}{*}{$\begin{array}{c}\text { Number } \\
\text { of in } \\
\text { silico } \\
\text { SNPs }\end{array}$} \\
\hline & \multirow{2}{*}{$\begin{array}{c}\text { Total } \\
\text { number }\end{array}$} & \multicolumn{3}{|c|}{ Length } & \multicolumn{2}{|c|}{$\begin{array}{l}\text { Do not overlap with } \\
\text { PSRs in pandemic } \\
\text { strains }\end{array}$} & \multirow{2}{*}{$\begin{array}{c}\text { Total } \\
\text { number }\end{array}$} & \multicolumn{3}{|c|}{ Length } & \multicolumn{2}{|c|}{$\begin{array}{l}\text { Do not overlap with } \\
\text { PSRs in non- } \\
\text { pandemic strains }\end{array}$} & \\
\hline & & Min & Max & Median & Number & $\begin{array}{l}\text { Percentage } \\
\text { of total, } \%\end{array}$ & & Min & Max & Median & Number & $\begin{array}{l}\text { Percentage } \\
\text { of total, \% }\end{array}$ & \\
\hline PB2 & 23 & 5 & 31 & 7 & 19 & 82.6 & 18 & 5 & 34 & 8.5 & 13 & 72.2 & 7 \\
\hline PB1 & 15 & 5 & 32 & 7 & 13 & 86.7 & 15 & 5 & 103 & 9 & 13 & 86.7 & 7 \\
\hline PA & 20 & 5 & 54 & 8 & 17 & 85.0 & 13 & 5 & 27 & 9 & 10 & 76.9 & 7 \\
\hline $\mathrm{HA}$ & 11 & 5 & 10 & 9 & 9 & 81.8 & 21 & 5 & 24 & 8 & 19 & 90.5 & 5 \\
\hline NP & 28 & 5 & 24 & 9 & 15 & 53.6 & 31 & 5 & 67 & 7 & 17 & 54.8 & 5 \\
\hline NA & 7 & 5 & 18 & 10 & 6 & 85.7 & 6 & 7 & 24 & 10.5 & 5 & 83.3 & 5 \\
\hline M1 & 22 & 5 & 57 & 10 & 10 & 45.5 & 15 & 5 & 30 & 8 & 2 & 13.3 & 2 \\
\hline M2 & 5 & 7 & 34 & 14 & 2 & 40.0 & 5 & 5 & 43 & 9 & 3 & 60.0 & 1 \\
\hline NS1 & 8 & 5 & 46 & 22.5 & 6 & 75.0 & 2 & 5 & 26 & 15.5 & 0 & 00.0 & 2 \\
\hline NS2 & 3 & 7 & 121 & 14 & 0 & 00.0 & 8 & 5 & 48 & 11 & 2 & 25.0 & 1 \\
\hline
\end{tabular}




\begin{tabular}{|l|l|l|l|l|l|l|l|l|l|l|l|l|}
\hline Total & 142 & 5 & 121 & 9 & 97 & 68.3 & 134 & 5 & 103 & 8 & 84 & 62.7 \\
\hline
\end{tabular}

\begin{tabular}{|c|c|c|c|c|c|c|c|c|}
\hline \multirow[t]{3}{*}{$\stackrel{\Perp}{\gtrless}$} & \multirow[t]{3}{*}{$\begin{array}{l}\text { Gene } \\
\text { name }\end{array}$} & \multirow[t]{3}{*}{$\begin{array}{l}\text { Total length } \\
\text { of PSRs }\end{array}$} & \multirow[t]{3}{*}{$\begin{array}{l}\text { Percentage of } \\
\text { mRNA } \\
\text { covered by } \\
\text { PSRs }\end{array}$} & \multicolumn{4}{|c|}{$\begin{array}{l}\text { Number of nucleotides within structured RNA } \\
\text { regions, which changed their probability of being } \\
\text { paired to a value outside of the naturally occurring } \\
\text { range of probabilities for this position }\end{array}$} & \multirow[t]{3}{*}{ P-value } \\
\hline & & & & \multicolumn{2}{|c|}{$\begin{array}{l}\text { Mutations in positions } \\
\text { that are prone to be } \\
\text { mutated }\end{array}$} & \multicolumn{2}{|c|}{$\begin{array}{l}\text { Mutations in conserved } \\
\text { positions }\end{array}$} & \\
\hline & & & & Mean & $\begin{array}{l}\text { Standard } \\
\text { deviation }\end{array}$ & Mean & $\begin{array}{l}\text { Standard } \\
\text { deviation }\end{array}$ & \\
\hline \multirow{10}{*}{ 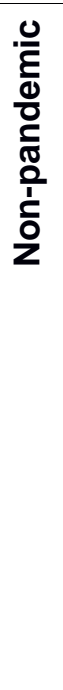 } & PB2 & 257 & 0.113 & 18.6 & 13.5 & 20.8 & 14.9 & 0.1395 \\
\hline & PB1 & 156 & 0.069 & 11.4 & 10.5 & 11.8 & 10.4 & 0.3264 \\
\hline & PA & 262 & 0.122 & 21.8 & 20.4 & 24.0 & 18.8 & 0.064 \\
\hline & $\mathrm{HA}$ & 85 & 0.050 & 5.9 & 5.4 & 5.3 & 5.6 & 0.0813 \\
\hline & NP & 300 & 0.200 & 24.6 & 17.2 & 31.3 & 17.7 & 0.001 \\
\hline & NA & 75 & 0.053 & 4.0 & 5.1 & 4.4 & 6.2 & 0.2283 \\
\hline & M1 & 334 & 0.440 & 24.3 & 23.3 & 21.1 & 21.2 & 0.1068 \\
\hline & M2 & 77 & 0.262 & 4.1 & 4.7 & 7.0 & 7.5 & 0.0022 \\
\hline & NS1 & 183 & 0.264 & 9.6 & 11.0 & 15.2 & 15.7 & 0.0008 \\
\hline & NS2 & 142 & 0.388 & 11.4 & 19.2 & 12.0 & 22.8 & 0.0311 \\
\hline$\stackrel{0}{\approx}$ & PB2 & 232 & 0.102 & 16.7 & 14.0 & 16.0 & 13.0 & 0.2883 \\
\hline
\end{tabular}




\begin{tabular}{|c|c|c|c|c|c|c|c|}
\hline PB1 & 251 & 0.110 & 15.8 & 14.3 & 17.0 & 14.6 & 0.1722 \\
\hline PA & 138 & 0.064 & 12.0 & 10.7 & 12.5 & 10.8 & 0.3317 \\
\hline HA & 205 & 0.121 & 17.5 & 18.0 & 15.9 & 18.5 & 0.0273 \\
\hline NP & 351 & 0.234 & 41.2 & 27.5 & 44.8 & 27.8 & 0.0955 \\
NA & 84 & 0.060 & 4.7 & 6.8 & 5.5 & 7.7 & 0.3006 \\
\hline M1 & 174 & 0.229 & 11.1 & 11.8 & 12.0 & 11.1 & 0.0917 \\
\hline M2 & 77 & 0.262 & 10.5 & 8.7 & 9.8 & 11.2 & 0.0395 \\
\hline NS1 & 31 & 0.047 & 2.8 & 5.7 & 3.3 & 5.7 & 0.1341 \\
\hline NS2 & 146 & 0.399 & 10.4 & 14.7 & 21.5 & 28.0 & 0.0007 \\
\hline
\end{tabular}


807 Table 3: List of antisense oligonucleotides used to examine influenza viral replication inhibition.

\begin{tabular}{|c|c|c|}
\hline Name & Sequence & Nucleotide position in NS2 mRNA \\
\hline stem & CAGAGACTCG & $105-114$ \\
\hline loop & TATATTTT & $115-122$ \\
\hline off10 & CTTATTTCCT & $221-230$ \\
rand10 & TATCCCACAC & NA \\
\hline
\end{tabular}

808

809

810

811

812

813

814

Figure 1: Computationally predicted by mfold 3.6 (71) (a) optimal and (b) one of many suboptimal secondary structures of tRNA. Mfold was used with the default energy parameters including the folding temperature fixed at $37^{\circ} \mathrm{C}$. Despite the fact that the left structure contains more base pairs, the right structure is functional and evolutionarily conserved.

Figure 2: Structure variability and mutability profiles for non-pandemic ((a), (b), and (c)) and pandemic ((d), (e), and (f)) NS2 influenza mRNAs. Plots (a) and (d) demonstrate structure conservation profiles; namely, they show the moving average that was calculated by applying a sliding window approach to smooth individual fluctuations of standard deviations of nucleotide base pairing probabilities. The blue solid line demonstrates the mean level of all moving average values, and the blue dashed line demonstrates the level equal to the mean of all moving average values decreased by the standard deviation of all moving average values. In this case, the mean and the standard deviation were computed based on all moving average values from all mRNAs of a particular type (pandemic or non-pandemic) of influenza strains. According to our definition, when the moving average goes below the blue dashed line, it is a structured RNA region. Such regions are colored with either yellow or green across the plots. Plots (b) and (e) demonstrate profiles of the mean values of probabilities of nucleotide positions to be in a double-stranded conformation. If this value is close to 1, it means that in most strains in the dataset the correspondent nucleotide has a very high probability to be paired; and, if this value is close to 0, the correspondent nucleotide is very likely to be unpaired in most strains in the dataset. Plots (c) and (f) demonstrate mutability profiles for NS2 mRNAs. Mutability of every nucleotide position is computed as a value of Shannon entropy which is calculated based on frequency of every ribonucleotide in a particular position. Areas within RNA colored with yellow or green demonstrate identified structured RNA regions. Meanwhile, areas colored with green show regions in which particular secondary structure was determined. 
829 Figure 3: Secondary structural elements identified in the NS2 mRNA of H1N1 influenza A virus. These structural elements are evolutionarily 830 conserved among analyzed strains. Hairpin at plot (b) was identified in both non-pandemic and pandemic H1N1 influenza. Structure shown at 831 plot (a) exists in non-pandemic influenza virus, while pandemic mRNAs contain only part of that structure covered by nucleotide positions 40 to 83273

834 Figure 4: Influenza viral replication inhibition effect of antisense oligonucleotides, 24 hours post infection. P-value for the comparison between 835 "Stem" and "Flu" is 0.0043 and is the only statistically significant difference. 
a.

3'

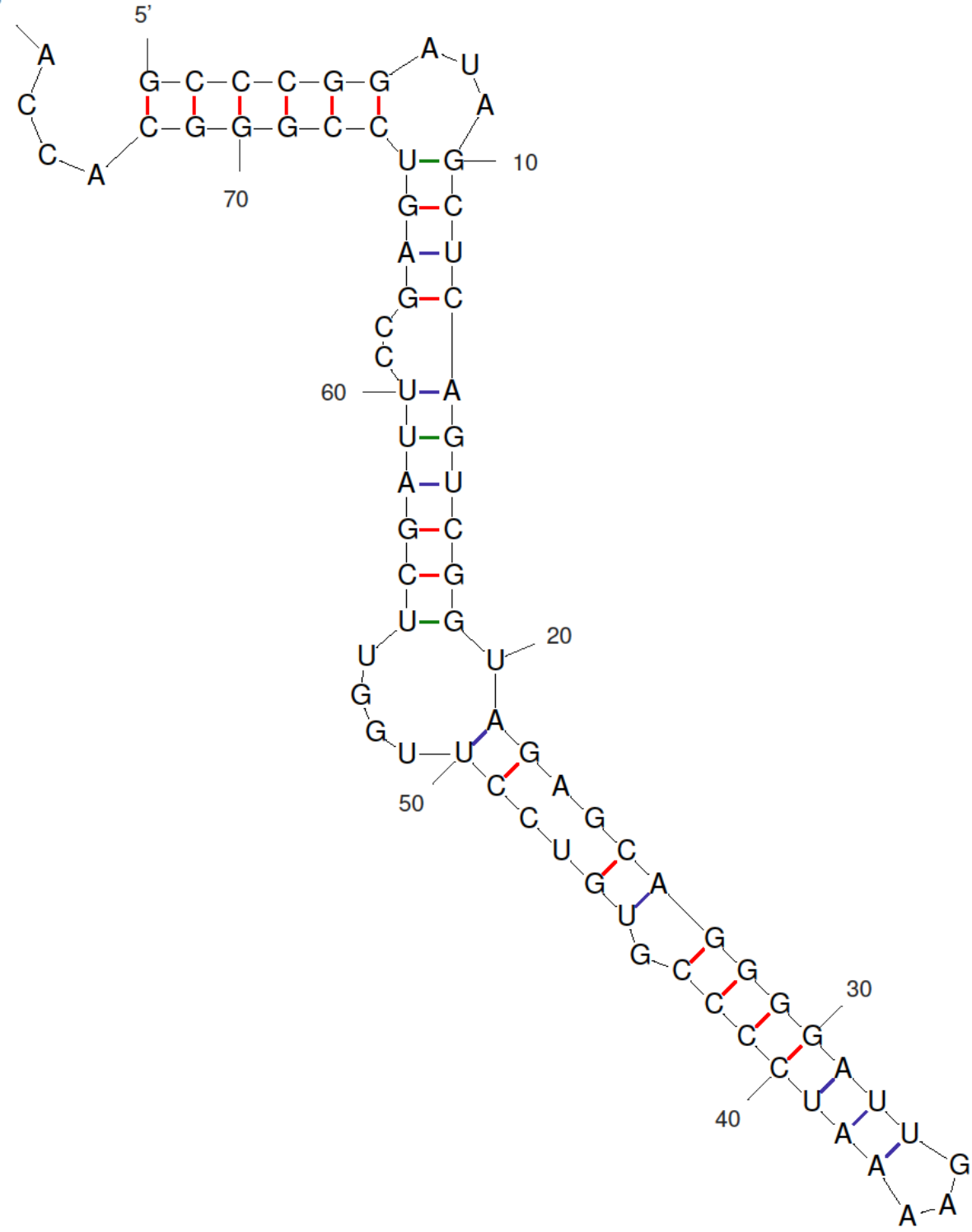

b.

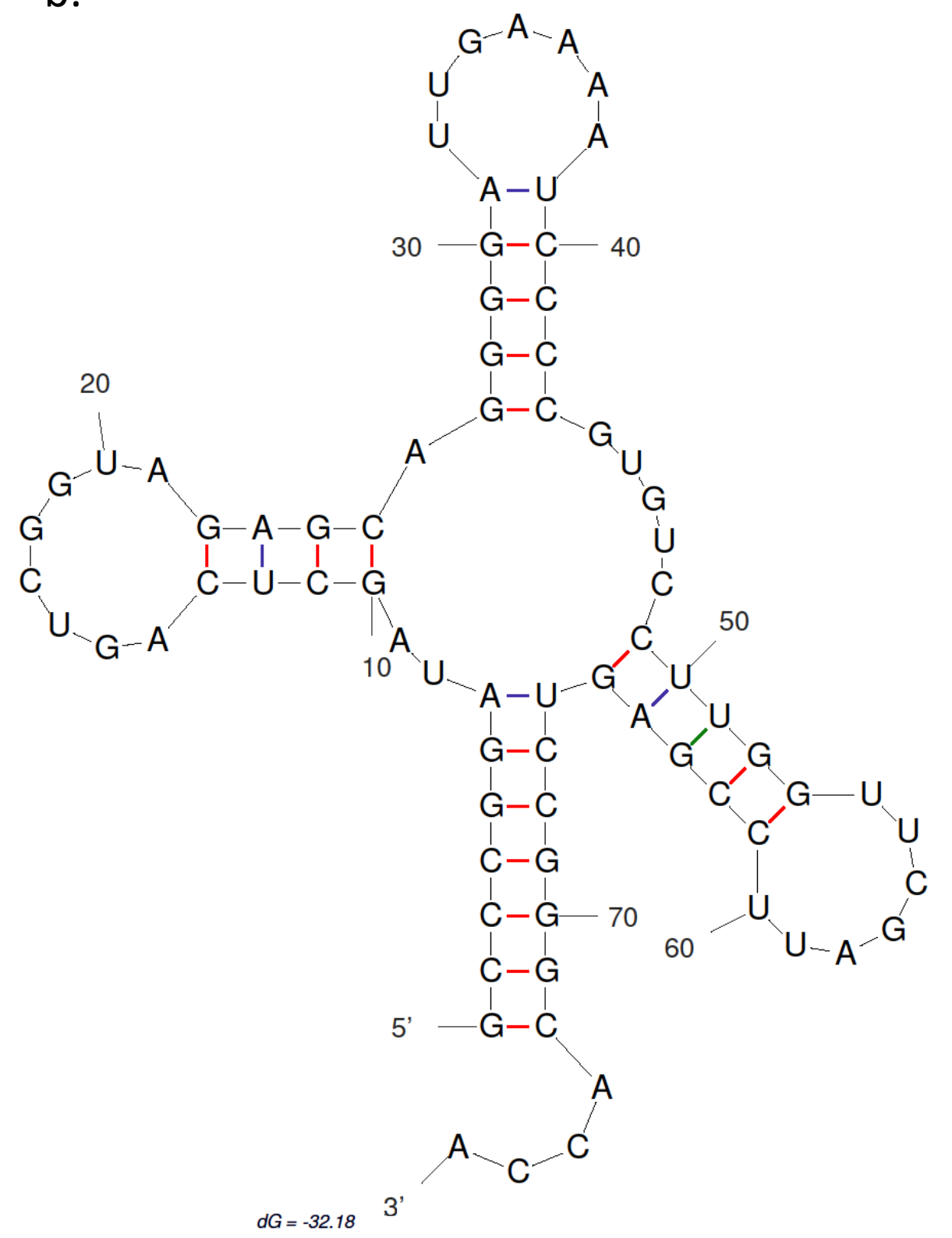


a.

NS2, non-pandemic d.

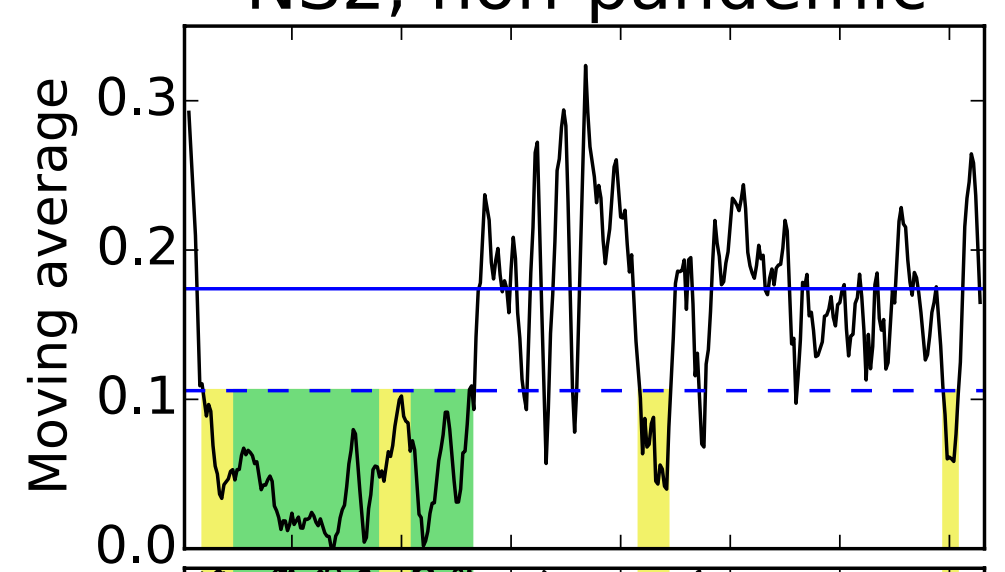

b.

$$
\begin{aligned}
& \begin{array}{ll}
\frac{0}{2} & 0.8 \\
\frac{\pi}{2} & 0.6
\end{array} \\
& \text { 당 } 0.4 \\
& \sum^{\infty} 0.2
\end{aligned}
$$

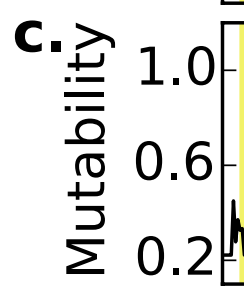

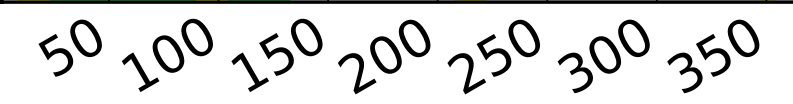
Length
NS2, pandemic

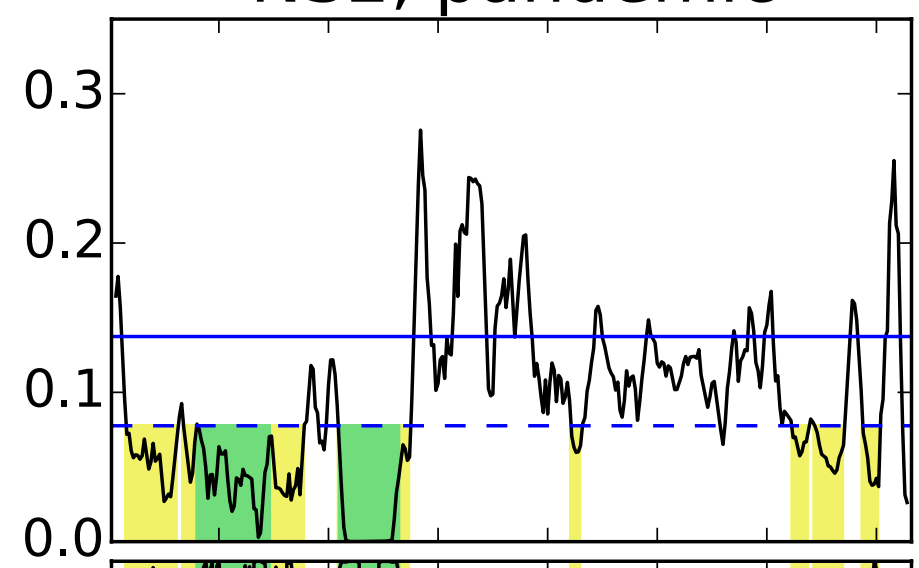

e.

0.8

0.6

0.4

0.2 .

,

f.

1.0
0.6
0.2

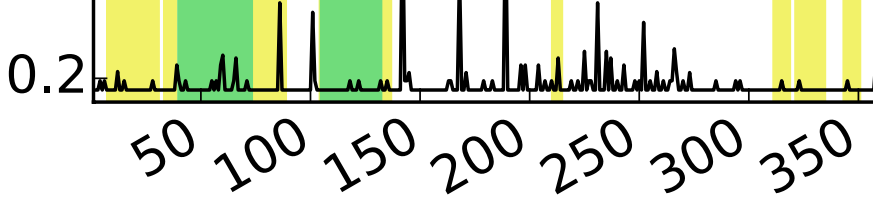
Length 


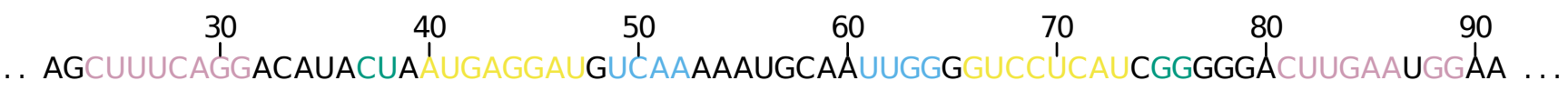

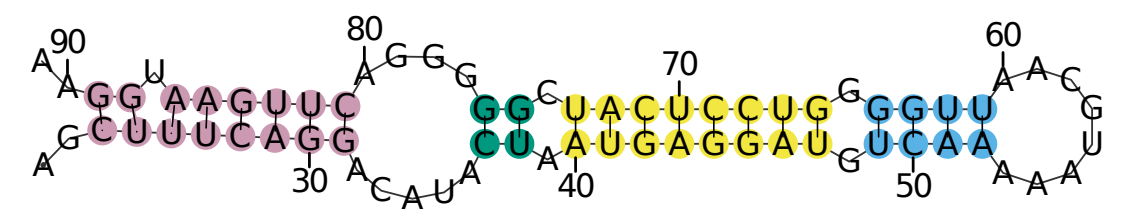

$\begin{array}{ccc}110 & 120 & 130 \\ \ldots . \text { UUCGAGUĆUCUGAAAAUÁUACAGAGAUU'CGCU ... }\end{array}$

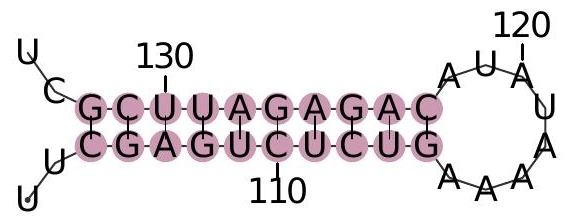




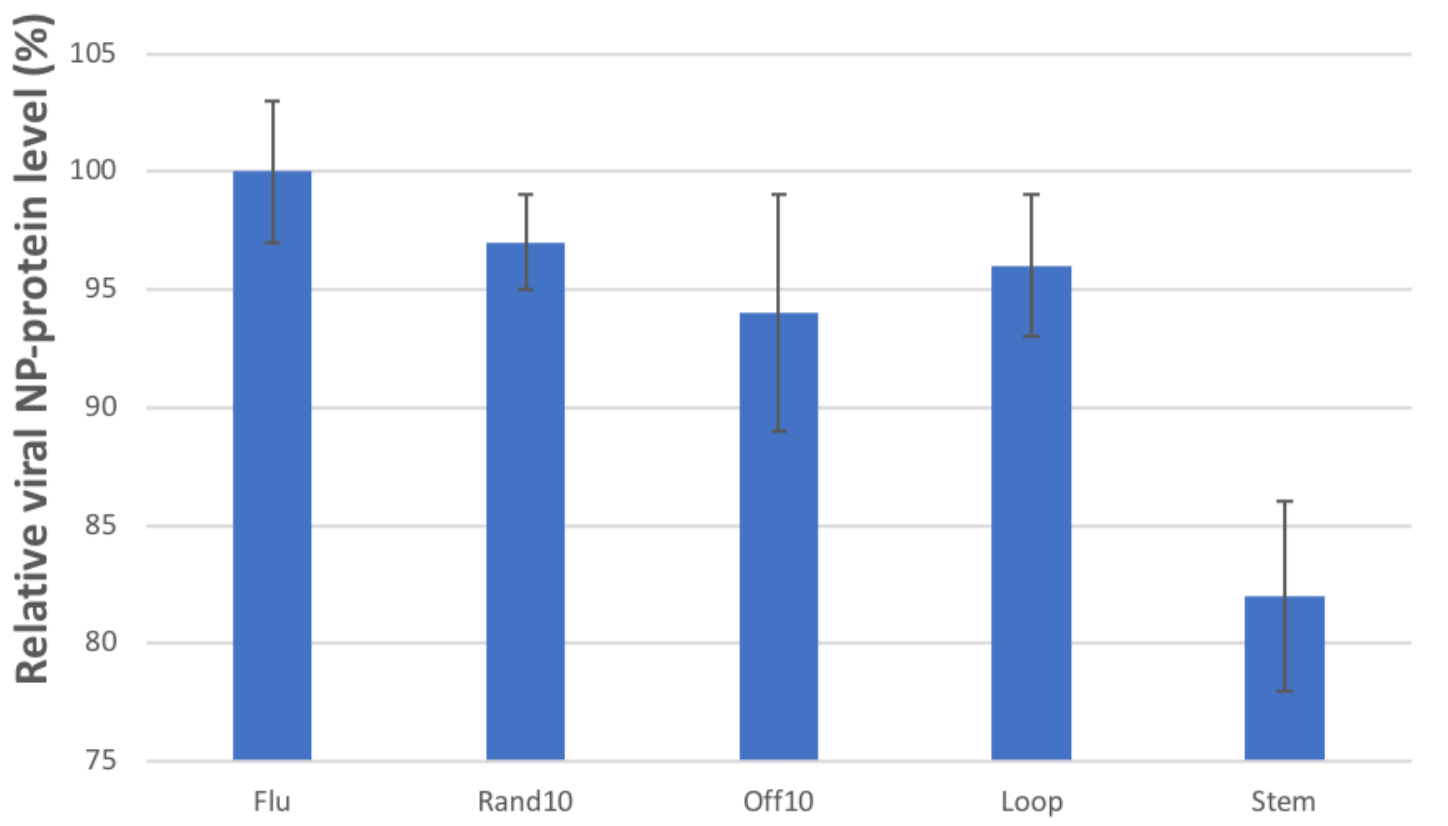

